\title{
The mental health experiences and needs of methamphetamine users in Cape Town: A mixed-methods study
}

\author{
M H Watt, ${ }^{1} \mathrm{PhD}$; B Myers, ${ }^{2} \mathrm{PhD} ; \mathrm{S}$ L Towe,,${ }^{1,3} \mathrm{PhD}$; C S Meade, ${ }^{1,3} \mathrm{PhD}$ \\ ${ }^{1}$ Duke University, Duke Global Health Institute, Durham, NC, USA \\ ${ }^{2}$ Alcohol, Tobacco and Other Drug Research Unit, South African Medical Research Council, Tygerberg, Cape Town, South Africa \\ ${ }^{3}$ Department of Psychiatry and Behavioral Sciences, Duke University School of Medicine, Duke Global Health Institute, Durham, NC, USA
}

Corresponding author: $M$ H Watt (melissa.watt@duke.edu)

\begin{abstract}
Background. South Africa (SA) has a burgeoning problem of methamphetamine use, particularly in the Western Cape Province. Although methamphetamine has been associated with elevated psychological distress, there has been little examination of the mental health needs of out-of-treatment methamphetamine users in SA.

Objective. To describe the mental health experiences and needs of out-of-treatment methamphetamine users in Cape Town.

Methods. Active methamphetamine users were recruited using respondent-driven sampling techniques. Eligible participants $(N=360)$ completed a computer-assisted assessment and clinical interview, where they provided data on mental health symptoms and treatmentseeking behaviour. A subset of 30 participants completed qualitative in-depth interviews in which they provided narrative accounts of their mental health experiences and needs. Analysis of the mixed-methods data was conducted using a concurrent triangulation strategy whereby both methods contributed equally to the analysis and were used for cross-validation.

Results. About half of the participants met screening criteria for depression and traumatic stress, and there were some indications of paranoia. Using substances to cope with psychological distress was common, with participants talking about using methamphetamine to numb their feelings or forget stressful memories. One-third of women and $13 \%$ of men had previously tried to commit suicide. Despite the huge mental health burden in this population, very few had ever received mental health treatment.

Conclusion. The data indicate a need for integrated care that addresses both substance use and psychiatric needs in this population. Mental health and drug treatment services targeting methamphetamine users should include a concerted focus on suicide prevention.

S Afr Med J 2015;105(8):685-688. DOI:10.7196/SAMJnew.7910
\end{abstract}

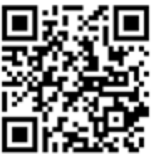

Since early 2000, there has been an exponential rise in the use of methamphetamine, locally called 'tik', in the Western Cape Province, South Africa (SA). Studies consistently show that methamphetamine use is associated with poor mental health, including depression, psychosis and suicidality. ${ }^{[1]}$ In SA, research has demonstrated an increased risk of mental health problems among methamphetamine users, including aggressive behaviour ${ }^{[2]}$ and symptoms of depression and anxiety. ${ }^{[3]}$

The relationship between methamphetamine and mental health is complex. On the one hand, poor mental health may be a risk factor for the initiation of substance use, as people may 'self-medicate' as a way to deal with psychiatric distress. ${ }^{[4]}$ On the other hand, substance use results in numerous psychosocial sequelae, including isolation, family breakdown and loss of employment, which can contribute to mental health problems. Chronic methamphetamine use is associated with structural and functional changes in the brain that may account for the increased rate of psychiatric disturbance in this population. ${ }^{[5]}$ Furthermore, the cycle of addiction (including intoxication, bingeing, withdrawal and craving) may lead to depressed mood, lethargy and anhedonia.

Regardless of whether psychiatric disturbances precede or follow the initiation of methamphetamine use, understanding the mental health experiences and needs of methamphetamine users in SA is important, as poor mental health may be a barrier to drug cessation and harm reduction efforts. Psychological distress impacts on motivation to initiate drug treatment services, ${ }^{[3]}$ and ongoing distress may contribute to attrition from treatment. In addition, mental health problems may serve as barriers to reducing harms such as risky sexual behaviours that are associated with continued substance use. Despite the potential impact of untreated mental disorders on treatment seeking and outcomes, there has been little research on how methamphetamine users experience symptoms of psychological distress. This gap needs to be addressed so that appropriate intervention programmes can be developed for methamphetamine users in SA.

\section{Methods \\ Overview}

The results presented here come from a mixed-methods study that included a cross-sectional survey of 360 current methamphetamine users and in-depth interviews (IDIs) with a subset of 30 participants. Data collection was completed between May and October 2013 in Delft, a township on the Cape Flats, Western Cape.

\section{Sample}

Participants were recruited into the cross-sectional survey using a respondent-driven sampling strategy, described elsewhere. ${ }^{[6]}$ Individuals were eligible to participate if they were $\geq 18$ years old and positive for methamphetamine use on a urine drug screen. A subset of 30 participants were selected to participate in IDIs to explore the study topics in greater depth. The IDI participants were purposefully selected to provide a balance of race and gender representation and to include individuals who the staff felt would be articulate and reflective. 


\section{Data collection}

\section{Quantitative procedures and measures}

Participants completed an audio computer-assisted self-interview, followed by a clinical interview. Data collection was conducted by trained staff in a private room. The visit took approximately 2 hours, and assessments were completed in the language of the participants' choice (Afrikaans, Xhosa or English).

Mental health symptoms. The nine-item Patient Health Questionnaire (PHQ-9) measured frequency of depressive symptoms in the past 2 weeks. Scores range from 0 to 27, and a dichotomous measure was created for moderate depression $(\geq 10) .{ }^{[7]}$ The seven-item Breslau Post-Traumatic Stress Disorder (PTSD) screener was used to capture traumatic stress symptoms in the past month. The yes/no responses were summed to create composite scores ranging from 0 to 7 ; a recommended cut-off of 4 was used as an indication of PTSD symptoms. ${ }^{[8]}$

Addiction Severity Index Lite (ASI-L). The ASI-L is a semistructured interview for substance-abusing populations that assesses several different areas of functioning, including substance use and mental health. ${ }^{[9]}$ Participants reported the number of days they had used methamphetamine in the past 30 days, as well as the number of years they had used methamphetamine regularly. They also reported the number of days they had experienced psychological problems in the past 30 days, whether they had ever experienced particular psychiatric symptoms, including hallucinations, suicidal ideation, suicide attempt and aggressive behaviour, and whether they had experienced these symptoms in the past 30 days. Participants were asked whether they had ever received treatment for psychological problems, whether they had ever been hospitalised for psychological problems, and whether they had ever been prescribed psychiatric medications. Participants rated how troubled they were by psychological symptoms and how important they perceived psychiatric treatment to be for them (with responses ranging from 'not at all' to 'extremely').

Substance use coping. Participants responded to the four-item substance use subscale of the $\mathrm{COPE}^{[10]}$ to assess how often they used substances as a coping mechanism. Response options ranged from 'never or rarely' to 'almost always.'

\section{Qualitative procedures}

The IDIs were conducted by local interviewers with extensive training in qualitative methods in the language of the participant's choice, and lasted approximately 60 - 90 minutes. A semistructured interview guide included broad opening questions and more specific follow-up probes. The mental health content included questions and probes about chronic and acute stressors, areas of emotional distress, distress symptoms including suicidal ideation and suicide attempts, coping behaviours, and any mental health treatment sought or received.

\section{Ethical concerns}

Given the sensitive nature of the interviews, the team received extensive and ongoing training by a local clinical psychologist on how to support participants, how and when to offer active referrals to local service agencies, and how to assess for risk of suicide. A list of referrals was provided to all participants, and the interviewer offered to make active referrals when participants were in distress. The psychologist was on call to advise staff on steps that needed to be taken with distressed participants. The study protocol was approved by ethical review boards at Stellenbosch University and Duke University.

\section{Analysis}

Analysis was conducted using a concurrent triangulation strategy, whereby qualitative and quantitative data contributed equally to the analysis and were used for cross-validation. For the quantitative analysis, descriptive statistics were used to characterise the mental health symptoms, needs and treatment-seeking behaviour of the sample. For the qualitative analysis, audio recordings of the interviews were transcribed and translated into English. Narrative memos were written to organise the content of the transcripts, make connections across the transcript content, and begin to achieve meaningful insights into the data. NVivo software was used to conduct content analysis. The quantitative and qualitative data were then combined to provide a full picture of the mental health experiences and needs of the population.

\section{Results}

The sample included 201 men and 159 women, described in Table 1. The 17 men and 13 women who completed IDIs had demographic and drug-related characteristics similar to the full sample.

\section{Presentation of mental health symptoms}

In the survey data, $49.3 \%$ of respondents met the criteria for moderate depression and $54.0 \%$ the screening criteria for PTSD. On average, individuals reported experiencing 11.7 days (range 0 - 30, standard deviation (SD) 12.8) of psychological problems in the past 30 days. Almost half of the sample (47.9\%) reported being 'extremely' bothered by psychological symptoms in the past month. Women reported more days of psychological problems than men (13.5 v. 10.2; $p<0.05$ ).

In the IDIs, most participants expressed psychological distress. Depressive symptoms included crying during the interview, loneliness, lethargy, and lack of interest in life. For men, feelings of regret and low self-worth were dominant, as this man expressed: 'My mind is suffering, because I had my own plans, but none of them are successful.' Several women talked about symptoms consistent with PTSD, typically related to histories of sexual or physical abuse. One woman, in recounting her sexual abuse history, said, 'When you are asking me about that I just feel like vomiting ... I don't want to talk about my difficult memories.'

Participants spoke about the impact of methamphetamine on their psychological state. Several said that methamphetamine made them more 'emotional'. One man stated that smoking methamphetamine 'puts you more in touch with your feelings. You are not $100 \%$ in control when it comes to thoughts or actions, but you are more emotional. You feel more. You sense more.' Hallucinations and paranoia also seemed to be common during periods of methamphetamine use When not intoxicated, people spoke about feeling extreme lethargy and lack of interest in life.

\section{Methamphetamine as a coping mechanism}

Using substances to cope with stressors was common, with nearly half of the survey sample saying that they used substances to feel better, lose themselves, avoid thoughts and get through difficult situations (Table 2). In the IDIs, participants spoke about methamphetamine as a 'comfort zone' and a way to 'calm down'. Many reported using methamphetamine to numb feelings, forget disturbing memories, and cope with their daily lives. A woman who had severe depression and a history of multiple traumas said that she used methamphetamine to cope with her emotional symptoms: 'The moment I increased my daily intake (of methamphetamine), my body felt much better. I could also manage emotionally'. A man spoke about how he used methamphetamine to deal with daily stressors: 'If the pressure gets too much, or I can't handle things, then I have a fix. I do that to cut myself off from everything and not focus. I just want to be narrow minded.' Five people specifically talked about initiating 
Table 1. Characteristics of the sample $(N=360)$

\begin{tabular}{|c|c|c|c|}
\hline & $\begin{array}{l}\text { Men } \\
(N=201)\end{array}$ & $\begin{array}{l}\text { Women } \\
(N=159)\end{array}$ & Statistic \\
\hline \multicolumn{4}{|l|}{ Demographics } \\
\hline Age, mean (SD) & $28.92(7.59)$ & $29.04(6.95)$ & $t(358)=0.15$ \\
\hline Race, coloured v. black African, $n(\%)$ & $125(62.2)$ & $138(86.8)$ & $\chi^{2}(1)=27.30^{* *}$ \\
\hline Currently married, $n(\%)$ & $22(10.9)$ & $28(17.6)$ & $\chi^{2}(1)=3.30$ \\
\hline Employed (part or full time), $n$ (\%) & $46(22.9)$ & $21(13.2)$ & $\chi^{2}(1)=5.49^{*}$ \\
\hline Completed secondary school, $n(\%)$ & $22(10.9)$ & $20(12.6)$ & $\chi^{2}(1)=0.23$ \\
\hline \multicolumn{4}{|l|}{ Methamphetamine use and addiction } \\
\hline Years of regular use, mean (SD) & $7.15(4.04)$ & $6.95(3.08)$ & $t(358)=0.52$ \\
\hline Days of use in past 30 days, mean (SD) & $24.26(8.73)$ & $22.52(9.05)$ & $t(358)=1.86$ \\
\hline \multicolumn{4}{|l|}{ Mental health } \\
\hline Depression symptoms (PHQ-9 $\geq 10$ ), $n$ (\%) & $96(47.8)$ & $81(50.9)$ & $\chi^{2}(1)=0.31$ \\
\hline PTSD symptoms (Breslau $\geq 4), n(\%)$ & $107(53.2)$ & $88(55.3)$ & $\chi^{2}(1)=0.11$ \\
\hline Days of problems, past 30 days, mean (SD) & $10.16(12.17)$ & $13.47(13.23)$ & $t(329)=2.37^{*}$ \\
\hline Felt extremely bothered by problems, $n$ (\%) & $89(44.3)$ & $78(49.1)$ & $\chi^{2}(1)=0.87$ \\
\hline Hallucinations, lifetime, $n(\%)$ & $104(51.7)$ & $63(39.6)$ & $\chi^{2}(1)=5.24^{*}$ \\
\hline Hallucinations, past 30 days, $n(\%)$ & $98(48.8)$ & $60(37.7)$ & $\chi^{2}(1)=4.38^{*}$ \\
\hline Suicidal ideation, lifetime, $n(\%)$ & $50(24.9)$ & $68(42.8)$ & $\chi^{2}(1)=12.90^{* *}$ \\
\hline Suicidal ideation, past 30 days, $n$ (\%) & $26(12.9)$ & $25(15.7)$ & $\chi^{2}(1)=0.57$ \\
\hline Suicide attempt, lifetime, $n$ (\%) & $26(12.9)$ & $53(33.3)$ & $\chi^{2}(1)=21.56^{\star *}$ \\
\hline Suicide attempt, past 30 days, $n(\%)$ & $9(4.5)$ & $16(10.1)$ & $\chi^{2}(1)=4.29^{*}$ \\
\hline Aggressive behaviour, lifetime, $n(\%)$ & $148(73.6)$ & $89(56.0)$ & $\chi^{2}(1)=12.31^{\star *}$ \\
\hline Aggressive behaviour, past 30 days, $n$ (\%) & $118(58.7)$ & $84(52.8)$ & $\chi^{2}(1)=1.25$ \\
\hline $\begin{array}{l}\text { Treatment perceived as extremely } \\
\text { important, } n(\%)\end{array}$ & $91(45.3)$ & $77(48.4)$ & $\chi^{2}(1)=0.44$ \\
\hline${ }^{* *} p<0.01,{ }^{*} p<0.05$ & & & \\
\hline
\end{tabular}

Table 2. Frequency of substance use as a coping mechanism to deal with stressors $(N=360)$

\begin{tabular}{lllll}
\hline & $\begin{array}{l}\text { Never/rarely } \\
\boldsymbol{n}(\%)\end{array}$ & $\begin{array}{l}\text { Occasionally } \\
\boldsymbol{n}(\%)\end{array}$ & $\begin{array}{l}\text { Often } \\
\boldsymbol{n}(\%)\end{array}$ & $\begin{array}{l}\text { Almost always } \\
\boldsymbol{n}(\%)\end{array}$ \\
\hline $\begin{array}{l}\text { Use alcohol or drugs to feel } \\
\text { better* }\end{array}$ & $37(10.3)$ & $132(36.7)$ & $123(34.2)$ & $67(18.6)$ \\
$\begin{array}{l}\text { Try to lose myself by drinking } \\
\text { or taking drugs }\end{array}$ & $50(13.9)$ & $151(41.9)$ & $97(26.9)$ & $62(17.2)$ \\
$\begin{array}{l}\text { Drink or take drugs to think } \\
\text { about it less }\end{array}$ & $49(13.6)$ & $138(38.3)$ & $108(30.0)$ & $65(18.1)$ \\
$\begin{array}{l}\text { Use alcohol or drugs to help } \\
\text { get through it } \\
\text { *One answer missing. }\end{array}$ & $60(16.7)$ & $145(40.3)$ & $97(26.9)$ & $58(16.1)$ \\
& & & & \\
\end{tabular}

methamphetamine for the first time as a way to cope with feelings or events in their lives.

\section{Suicidality}

In the survey data, $32.8 \%$ of the sample said that they had ever had suicidal thoughts, and $21.9 \%$ said that they had ever attempted suicide. Women were significantly more likely to have ever had suicidal thoughts $(42.8 \% \mathrm{v}$.
$24.9 \% ; p<0.001)$ and to have ever attempted suicide ( $33.3 \%$ v. $12.9 \%$; $p<0.001)$. In the IDIs, 10 of the 30 participants recounted suicide attempts, with many of those individuals reporting multiple attempts. The reasons that men expressed for attempting suicide were feeling rejected by family as a result of drug use, breaking up with a girlfriend, and feeling in a 'dark place'. Among women, four attributed their suicide attempts to traumas (sexual abuse, partner abuse and husband's murder), and the others spoke about general distress and problems in relationships. The most common way that people attempted suicide was via medication overdose, followed by hanging and cutting. Suicidal ideation was also common, with participants talking about feeling that they would be 'better off dead'.

\section{Treatment-seeking behaviour}

Only $7.2 \%$ of the survey sample had ever received treatment for a psychological problem. The majority (69.2\%) of those who reported that that they had received treatment said that they had been hospitalised for psychiatric reasons. Despite low uptake of mental health treatment, almost half (47.1\%) said that receiving psychological treatment was 'extremely important' to them. In the qualitative data, there was little discussion of mental health treatment. Five participants said that they had received some psychiatric treatment after being hospitalised for a suicide attempt, but none had follow-up after discharge. One woman explained that she felt that providers were not interested in treating drug users: 'After I came back from the hospital [after suicide attempt], they said they would check on me sometimes, but they never did. I told you before, not everyone is interested in us.' Only one of the IDI participants had received outpatient mental health counselling, which she apparently received through the HIV clinic where she was receiving care. Notably, several participants spoke about the study contact as a therapeutic experience, indicating a desire and need for mental health counselling. As this woman explained: 'I am feeling a great relief after talking to you. A heavy weight is lifted from my shoulders.'

\section{Discussion}

While previous studies in SA have documented the high prevalence of mental health problems among methamphetamine users, ${ }^{[2,3]}$ none have explored how users understand the relationship between methamphetamine use and psychological distress. We combined quantitative and qualitative methods to document the mental health experiences and needs of out-oftreatment methamphetamine users from one township in Cape Town. This study yields information that could potentially inform the development of mental health services for methamphetamine users in SA.

We found high rates of psychological distress in this population, with close to half of the participants scoring above the cut-off for depression and more than half meeting screening criteria for PTSD. Despite the high 
burden of mental disorders, very few participants had ever accessed mental health services, indicating a large unmet need for mental health treatment. This is not surprising, given evidence of a $75 \%$ treatment gap for common mental disorders in SA. ${ }^{[1]]}$ Further complicating matters for a substance-using population, substance abuse and other mental health services are delivered by distinct service systems, with neither system equipped to address both problems effectively in an integrated manner, ${ }^{[12]}$ although our findings indicate that a substantial proportion of methamphetamine users could benefit from integrated substance abuse and mental health services. Some might argue that the depressive symptomatology in this population is likely to be a consequence of the physiological effects of methamphetamine ${ }^{[5]}$ and may resolve after a period of abstinence. However, this explanation does not account for the high rates of PTSD observed in this sample. Our qualitative data suggest that traumatic experiences are likely to have preceded the initiation of methamphetamine use, with many participants reporting that they used methamphetamine as a way of coping with negative feelings associated with these experiences. For these participants, effective treatment requires addressing their traumatic experiences, particularly as unresolved trauma is associated with poor drug treatment outcomes. ${ }^{[13]}$

In addition to using methamphetamine as a way of coping with traumatic experiences, participants reported using methamphetamine to handle their everyday stressors. This is likely to be the result of poor problem-solving and coping skills. We also observed gender differences in how methamphetamine use was used to help people cope with stressful events. Women's narratives suggest that methamphetamine is used as an avoidance strategy to block, numb or dull negative psychological distress. In contrast, men reported using methamphetamine to facilitate emotional discharge or catharsis, a form of emotion-focused coping. The reliance on these maladaptive coping styles highlights the need for interventions to help methamphetamine users develop more adaptive problemfocused coping strategies.

Addressing psychological distress is particularly important in view of the alarmingly high rates of suicide attempts in this population. More than a third of women and more than $10 \%$ of men reported prior suicide attempts, higher than the national prevalence of $3 \%{ }^{[14]}$ This finding provides further evidence of the need to integrate mental health services into existing substance abuse programmes. At the very least, all patients reporting methamphetamine use should be screened for risk of suicide, and programming for methamphetamine dependence should include a focus on suicide prevention.

These findings have several other implications for the prevention and treatment of methamphetamine use. First, universal prevention programmes should consider equipping young people with problem-focused coping skills to help delay initiation of substance use. Second, prevention programmes indicated for individuals who are using methamphetamine but are not yet dependent should include a focus on developing adaptive coping and problemsolving styles, particularly given emerging evidence that brief, problem-solving therapies are effective for reducing substance use involvement in high-risk SA populations. ${ }^{[15]}$ Third, as mental health problems and problem-solving deficits are risk factors for poor treatment outcomes, ${ }^{[15]}$ substance abuse services should screen all methamphetamine users for co-occurring mental health problems and provide patients with coping and problem-solving interventions to help them deal with life stressors. Finally, the low use of mental health services in this population indicates a need for improving community awareness of mental health problems and resources, which may hinder uptake and consistent use of available community-based services.

\section{Study strengths and limitations}

While this study has several strengths, including the largest sample of community-recruited methamphetamine users in SA, it does have some limitations. First, findings may not generalise to the entire population of methamphetamine users or to methamphetamine users in other communities. Second, our sample may have been skewed towards regular methamphetamine users, as we only included participants with a positive urine screen that detects methamphetamine in the urine for 3 - 5 days. Although we may have excluded less frequent users, our results do reflect the need for mental health services among regular methamphetamine users. Finally, we did not undertake clinical interviewing to determine mental health diagnoses, but rather relied on symptom checklists.

\section{Conclusion}

This study clearly demonstrates that mental health services should be made more readily available to methamphetamine users. The high prevalence of suicide attempts and psychological distress in this sample highlights the need for evidence-based substance abuse prevention interventions that also promote mental health through developing adaptive coping strategies. Additionally, to improve drug treatment outcomes, programmes should screen methamphetamine users for suicide risk and psychological distress, deliver services that treat their substance use and underlying mental health conditions in an integrated manner, and teach adaptive coping strategies to prevent relapse to substance use.

Acknowledgements. This study was funded by the National Institute of Drug Abuse (R03-DA033282) and further supported by additional training and institutional grants from the National Institutes of Health (K23-DA028660, T32-AI007392, P30-AI064518, R25-DA035692), USA. We are grateful to all the men and women who participated, and to our collaborators and staff in SA (Donald Skinner, Desiree Pieterse, Albert Africa, Tembie Mafikizolo, and Mariana Bolumbe).

\section{References}

1. Marshall BD, Werb D. Health outcomes associated with methamphetamine use among young people: A systematic review. Addiction 2010;105(6):991-1002. [http://dx.doi.org/10.1111/j.13600443.2010.02932.x]

2. Pluddemann A, Flisher AJ, McKetin R, Parry C, Lombard C. Methamphetamine use, aggressive behavior and other mental health issues among high-school students in Cape Town, South Africa. Drug Alcohol Depend 2010;109(1-3):14-19. [http://dx.doi.org/10.1016/j.drugalcdep.2009.11.021]

Myers B, Kline TL, Doherty IA, Carney T, Wechsberg WM. Perceived need for substance use treatment Myers B, Kline TL, Doherty IA, Carney T, Wechsberg WM. Perceived need for substance use treatment
among young women from disadvantaged communities in Cape Town, South Africa. BMC Psychiatry among young women from disadvantaged communities in

4. Robinson J, Sareen J, Cox BJ, Bolton J. Self-medication of anxiety disorders with alcohol and drugs: Results from a nationally representative sample. J Anxiety Disord 2009;23(1):38-45. [http://dx.do. org/10.1016/j.janxdis.2008.03.013]

5. Rusyniak DE. Neurologic manifestations of chronic methamphetamine abuse. Psychiatr Clin North Am 2013;36(2):261-275. [http://dx.doi.org/10.1016/j.psc.2013.02.005]

6. Kimani SM, Watt MH, Merli MG, et al. Respondent driven sampling is an effective method for engaging methamphetamine users in HIV prevention research in South Africa. Drug Alcohol Depen 2014;143:134-140. [http://dx.doi.org/10.1016/j.drugalcdep.2014.07.018]

7. Kroenke K, Spitzer RL, Williams JB. The PHQ-9: Validity of a brief depression severity measure. J Gen Intern Med 2001;16(9):606-613. [http://dx.doi.org/10.1046/j.1525-1497.2001.016009606.x]

8. Breslau N, Peterson EL, Kessler RC, Schultz LR. Short screening scale for DSM-IV posttraumatic stress Breslau N, Peterson EL, Kessler RC, Schultz LR.
disorder. Am J Psychiatry 1999;156(6):908-911.

McLellan AT, Luborsky L, Woody GE, O'Brien CP. An improved diagnostic evaluation instrument for substance abuse patients. The Addiction Severity Index. J Nerv Ment Dis 1980;168(1):26-33.

substance abuse patients. The Addiction Severity Index. J Nerv Ment Dis 1980;168(1):26-33.
10. Carver CS, Scheier MF, Weintraub JK. Assessing coping strategies: a theoretically based approach. J Carver CS, Scheier MF, Weintraub JK.
Pers Soc Psychol 1989;56(2):267-283.

11. Seedat S, Stein DJ, Herman A, et al. Twelve-month treatment of psychiatric disorders in the South African Stress and Health Study (World Mental Health Survey Initiative). Soc Psychiatry Psychiat Epidemiol 2008;43(11):889-897. [http://dx.doi.org/10.1007/s00127-008-0399-9]

12. Myers B, Fakir N. Provision of Mental Health Services in South African Substance Abuse Treatmen Facilities. Int J Ment Health Addict 2009; 7(3):441-449. [http://dx.doi.org/10.1007/s11469-009-9205-5]

3. Norman SB, Tate SR, Anderson KG, Brown SA. Do trauma history and PTSD symptoms influence addiction relapse context? Drug Alcohol Depend 2007;90(1):89-96. [http://dx.doi.org/10.1016/j. drugalcdep.2007.03.002]

14. Joe S, Stein DJ, Seedat S, Herman A, Williams DR. Non-fatal suicidal behavior among South Africans: Results from the South Africa Stress and Health Study. Soc Psychiatry Psychiatr Epidemiol 2008;43(6):454-461. [http://dx.doi.org/10.1007/s00127-008-0348-7]

15. Sorsdahl K, Myers B, Ward CL, et al. Adapting a blended motivational interviewing and problemsolving intervention to address risky substance use amongst South Africans. Psychother Res 2015;25(4):435-444. [http://dx.doi.org/10.1080/10503307.2014.897770] 